\title{
Anesthesia in patients with arthrogryposis multiplex congenita: a report of 10 patients
}

\author{
Jae Woong Jung, Burn Young Heo, Eun Jung Oh, and Yang Hoon Chung \\ Department of Anesthesiolgy and Pain Medicine, Samsung Medical Center, Sungkyunkwan University School of Medicine, Seoul, \\ Korea
}

Arthrogryposis multiplex congenita (AMC) is a rare clinical syndrome characterized by congenital, non-progressive, multiple and persistent joint contractures with generalized muscle wasting. Patients with AMC undergo frequent anesthetic care in the correction of musculoskeletal deformities or other associated anomalies [1]. We retrospectively reviewed our perioperative experience with 10 patients with AMC from 2005 to 2013 in a single center. These patients underwent a total of 31 inductions of general anesthesia, of which 28 cases were for orthopedic surgeries. Each patient received multiple exposures to anesthesia, from one to 10 times.

In 25 anesthetic cases, general anesthesia was induced with thiopental and maintained with inhalation anesthetics. In another six cases, induction was performed with total intravenous anesthesia (TIVA) using propofol and remifentanil. The reasons for adopting TIVA were various: 1) Patients had mitochondrial myopathy (2 patients, 3 operations), 2) To allow neurologic monitoring during surgery to correct scoliosis (1 patient, 1 operation), and 3) To prevent malignant hyperthermia $(\mathrm{MH})$ according to the anesthesiologist's preference (2 patients, 2 operations).

Difficult intubation was observed in one patient. The patient was a 3-year-old girl in a bedridden state due to muscle weakness. Her weight was $8.5 \mathrm{~kg}$. She was scheduled for orthopedic correction of club foot. She had micrognathia and a high-arched palate, but no cervical vertebral anomalies. The opening of the patient's mouth was very narrow (2 fingers). Under direct laryngoscopy, the vocal cords were located anteriorly and obscured by excessive secretions. After oral suction, intubation was successfully performed with a stylet by an expert anesthesiologist.
The patient's laryngeal grade was III according to the CormackLehane scoring system.

There were nine cases of intraoperative hyperthermia in which patients' temperatures rose above $37.5^{\circ} \mathrm{C}$. In every case but one, the temperatures were restored to the normal range by conservative treatments such as cooling the operating room and turning off the warming blanket. In one case, a 4-year-old boy was presumed to be experiencing $\mathrm{MH}$. The patient's associated anomalies included osteochondromatosis, developmental delay, and mitochondrial myopathy. He weighed $16.8 \mathrm{~kg}$ and was scheduled for orthopedic surgery for hip joint and lower leg abnormalities. General anesthesia was induced with TIVA. Thirty minutes after induction, the patient's heart rate gradually increased from 100 to 180 beats per minute (bpm). Simultaneously, his temperature rose from 36.5 to $37.2^{\circ} \mathrm{C}$. When the patient's temperature reached $37.2^{\circ} \mathrm{C}$, we turned the warming blanket off and lowered the temperature of the operating room. However, the patient's body temperature continued to rise, to a maximum of $38.1^{\circ} \mathrm{C}$. As $\mathrm{MH}$ had not been completely ruled out, dantrolene was injected intravenously. Following the injection, the patient's heart rate decreased to $140 \mathrm{bpm}$ and his temperature decreased from 38.1 to $37.5^{\circ} \mathrm{C}$. After the conclusion of the operation, in the intensive care unit, the patient's heart rate again increased to $160-190 \mathrm{bpm}$ and his body temperature rose to $38.4^{\circ} \mathrm{C}$. In response, the pediatrician ordered dantrolene $17 \mathrm{mg}$ to be given to the patient as an intravenous injection four times a day from postoperative day (POD) 0 . After the second injection of dantrolene, the patient's heart rate decreased to $120-140 \mathrm{bpm}$, but his high fever persisted. On POD 2, the patient's body tempera-

Corresponding author: Burn Young Heo, M.D., Ph.D., Department of Anesthesiology and Pain Medicine, Samsung Medical Center, Sungkyunkwan University School of Medicine, 81, Irwon-ro, Gangnam-gu, Seoul 135-710, Korea. Tel: 82-2-3410-0736, Fax: 82-2-3410-6626, E-mail: burnyoung.heo@samsung.com

() This is an open-access article distributed under the terms of the Creative Commons Attribution Non-Commercial License (http:// creativecommons.org/licenses/by-nc/3.0/), which permits unrestricted non-commercial use, distribution, and reproduction in any medium, provided the original work is properly cited. 
Table 1. Associated Anomalies in 10 Patients with Arthrogryposis Multiplex Congenita

\begin{tabular}{lc}
\hline \multicolumn{1}{c}{ Anomalities } & Number of patients \\
\hline Club foot & 3 \\
Talus anomaly & 2 \\
Hip joint anomaly & 3 \\
Upper arm anomaly & 2 \\
Scoliosis & 2 \\
Inguinal hernia & 1 \\
Cerebral palsy & 1 \\
Small mandible & 2 \\
High arched palate & 1 \\
Cryptorchism & 1 \\
Funnel chest & 1 \\
Mitochondrial myopathy & 2 \\
\hline
\end{tabular}

ture decreased to normal. Although the case did not completely fit the criteria for $\mathrm{MH}$, we had a clinical impression of $\mathrm{MH}$ and treated the patient with injections of dantrolene, which appeared to be effective. Fortunately, most cases of anesthesia finished uneventfully, except for the several cases mentioned above. Associated anomalies of the patients are summarized in Table 1.

AMC is usually associated with multiple congenital anomalies, such as skeletal deformities, congenital heart disease, and abnormalities of the respiratory and genitourinary systems. The problems faced by anesthesiologists in dealing with patients with this syndrome include difficult airway, $\mathrm{MH}$ associated with myopathy, cardiorespiratory problems, difficult regional blockade, difficult IV access, and difficult positioning [2].

The most important issue is the possibility of difficult intubation. The most common maxillofacial findings in patients with AMC include decreased mandibular opening, micrognathia, a high arched palate, and deficient musculature of the orofacial complex. For these reasons, direct laryngoscopy or tracheal intubation may be difficult or impossible. There are many reports detailing how these problems were overcome with the use of LMAs, fiberoptic intubation, or fiberoptic-guided intubation via the LMA [3]. Postoperative airway problems are also important. Patients with AMC are more susceptible to respiratory depression due to associated anomalies such as myopathy, pulmonary hypoplasia, and thoracic and spine deformities. Patients with AMC are also vulnerable to perioperative aspiration because of loss of normal muscle movement and gastroesophageal reflux.

Another concern for anesthesiologists is MH. It is controversial whether AMC is associated with $\mathrm{MH}$ or not [4]. However, several myopathies themselves have strong relations to $\mathrm{MH}$. About $8 \%$ of the etiology of AMC is related to neuromuscular disorders, myopathic processes such as congenital muscular dystrophies, mitochondrial disturbances, or abnormalities of the biochemistry of the muscles [5]. In patients displaying symptoms of $\mathrm{MH}$, the Association of Anaesthetists of Great Britain \& Ireland has recommended dantrolene injection $2.5 \mathrm{mg} / \mathrm{kg}$ immediately, and repeat $1 \mathrm{mg} / \mathrm{kg}$ boluses as required to a maximum of $10 \mathrm{mg} / \mathrm{kg}$, until the improvement of symptoms. We encountered one suspected case of MH despite the use of TIVA. Evidently non-triggering anesthetics cannot completely prevent the occurrence of $\mathrm{MH}$. Therefore, anesthesiologists should be aware of the risk of $\mathrm{MH}$ in patients with AMC and should closely monitor these patients.

In addition, multiple contractures of the extremities make it difficult to maintain intraoperative positioning. Careful attention is required and appropriate measures, such as padding for reducing compression pressure, must be taken. Moreover, peripheral IV access is difficult in these patients because of tense, glossy skin, and scanty subcutaneous tissues.

In summary, the patient with AMC needs meticulous anesthetic care during corrective surgery. Various associated anomalies, rather than AMC itself, pose challenges to anesthesiologists. The anesthesiologists should consider not only the joint problems but also the associated anomalies - cardiac, gastrointestinal, genitourinary, respiratory, myopathic, neurogenic, and facial anomalies. In our cases, most patients with AMC experienced no adverse events during surgery. The most important concerns are the possibilities of difficult airway management and hyperthermia, especially combined with myopathies. Careful airway evaluation, perioperative monitoring of body temperature, tachycardia, and hypercarbia, and preparation for emergencies are needed to avoid critical events.

\section{References}

1. Martin S, Tobias JD. Perioperative care of the child with arthrogryposis. Paediatr Anaesth 2006; 16: 31-7.

2. Pujari VS, Shivanna S, Anandaswamy TC, Manjunath AC. Arthrogryposis multiplex congenita: An anesthetic challenge. Anesthesia Essays and Researches 2012; 6: 78-80.

3. Thomas PB, Parry MG. The difficult paediatric airway: a new method of intubation using the laryngeal mask airway, Cook airway exchange catheter and tracheal intubation fibrescope. Paediatr Anaesth 2001; 11: 618-21.

4. Baines DB, Douglas ID, Overton JH. Anaesthesia for patients with arthrogryposis multiplex congenita: what is the risk of malignant hyperthermia? Anaesth Intensive Care 1986; 14: 370-2.

5. Bonilla-Musoles F, Machado LE, Osborne NG. Multiple congenital contractures (congenital multiple arthrogryposis). J Perinat Med 2002; 30: 99-104. 